\title{
Influence of Crystallographic Orientation on the Response of Copper Crystallites to Nanoindentation
}

\author{
Aleksandr V. Korchuganov ${ }^{1, \text { a) }}$, Dmitrij S. Kryzhevich ${ }^{1,2, b)}$, \\ Konstantin P. Zolnikov ${ }^{1,2, c)}$, and Sergey G. Psakhie ${ }^{3,4, d)}$ \\ ${ }^{1}$ Institute of Strength Physics and Materials Science SB RAS, Tomsk, 634055, Russia \\ ${ }^{2}$ National Research Tomsk State University, Tomsk, 634050, Russia \\ ${ }^{3}$ National Research Tomsk Polytechnic University, Tomsk, 634050, Russia \\ ${ }^{4}$ Skolkovo Institute of Science and Technology, Skolkovo, 143025, Russia \\ a) Corresponding author: avkor@ispms.tsc.ru \\ b) kryzhev@ispms.tsc.ru \\ c) kost@ispms.tsc.ru \\ ${ }^{\mathrm{d})} \mathrm{sp@ispms.tsc.ru}$
}

\begin{abstract}
Molecular dynamics simulation was performed to study the features of nucleation and development of plastic deformation in copper crystallites in nanoindentation with different crystallographic orientations of their loaded surface: $(01 \overline{1}),(001)$, and (111). Atomic interaction was described by a potential constructed in terms of the embedded atom method. It is shown that behavior of the crystallite reaction force correlates well with a change in the fraction of atoms involved in local structural rearrangements. The generation of local structural changes decreases the slope of the crystallite reaction force curve or results in an extremum due to internal stress relaxation. Analysis of structural changes in the material being indented demonstrates that the orientation of its loaded surface greatly affects the features of nucleation and development of plastic deformation.
\end{abstract}

Keywords: plastic deformation, local structural changes, copper, molecular dynamics

\section{INTRODUCTION}

Now the production of various micro- and nanoelectromechanical systems is being actively developed. Many components of such systems while in operation are in mechanical contact with each other. Understanding the mechanisms of their interaction on the atomic scale is important for improvement of mechanical properties of available systems and materials and for development of new ones. Research in the properties and behavior of nanosized objects requires reliable and precise techniques. One of the most efficient and widely used methods for studying the physicomechanical properties of nanostructured materials and thin films is nanoindentation $[1,2]$. The method is based on local loading of material and simultaneous recording of its deformation response. Along with experimental research, computer simulation of the material behavior during nanoindentation has been actively developed in the last decades [3-6]. One of the significant advantages of computer simulation is the possibility of real time research in atomic structures and peculiarities of microscale processes in materials. In the context of the foregoing, the objective of the present work is to study the features of nucleation and development of plastic deformation in copper crystallites during nanoindentation on the atomic scale with different crystallographic orientations of their loaded surface.

\section{SIMULATION DETAILS}

The materials under study were fcc copper crystallites. Atomic interaction was described by a potential [7] constructed in terms of the embedded atom method [8]. The potential allows highly accurate description of structural

International Conference on Physical Mesomechanics of Multilevel Systems 2014
AIP Conf. Proc. 1623, 295-298 (2014); doi: 10.1063/1.4898940

(C) 2014 AIP Publishing LLC 978-0-7354-1260-6/\$30.00 
peculiarities of free surfaces, energy parameters of point defects and their complexes, elastic characteristics of copper crystallites, etc. The model crystallites were cubes with a side of $165 \AA$ and consisted of 350000 atoms. It should be noted that simulation results depend largely on the crystallite structure, crystallographic orientation, indenter shape, loading pattern, boundary conditions, etc. The indenters often used in calculations and experiments have spherical or pyramidal shapes. They form a quasi-point contact zone in a crystallite. Nucleation and development of plastic deformation in this zone is rather difficult to analyze. For better descriptiveness of structural changes in the crystallites and simplification of simulation data analysis, an absolutely rigid extended indenter in the form of a semicylinder consisting of copper atoms was used. With this indenter, the contact region is linearly extended from one crystallite face to the other. The indenter axis was parallel to the loaded crystallite surface. Along this axis, periodic boundary conditions were modeled. The loaded face was a free surface, whereas several atomic layers of the crystallite face opposite to it were prohibited to displace in the indentation direction. The lateral crystallite faces were modeled as free surfaces. Calculations were performed for three loaded surface orientations: $(01 \overline{1}),(001)$, and (111). The indenter velocity was $50 \mathrm{~m} / \mathrm{s}$; the crystallite temperature was $300 \mathrm{~K}$. The influence of loaded surface orientation on nucleation of structural defects was studied through calculations of dependences of the crystallite reaction force $P$ and fraction of atoms $\mathrm{n}$ involved in local structural changes on the indenter penetration depth $h$. Local structural changes were identified by common neighbor analysis $[9,10]$.

\section{SIMULATION RESULTS}

The curves $P(h)$ and $n(h)$ calculated for the loaded surface orientations under study are shown in Fig. 1. Open circles on the curves $P(h)$ indicate the indenter penetration depths at which abrupt changes in the slope of the loading curve or clearly defined breaks are observed. It is found that the peculiarities of the curves $P(h)$ and $n(h)$ in all three cases correlate well with each other. In particular, calculations show that generation of local structural changes decreases the slope of the curve $P(h)$ or results in an extremum due to internal stress relaxation. Projections of the crystallite structure for three loaded surface orientations at an indenter penetration depth of $10.0 \AA$ are presented in Fig. 2.

\section{Surface $(01 \overline{1})$}

The dependences of the reaction force and fraction of atoms involved in local structural changes on the indenter penetration depth for the loaded surface $(01 \overline{1})$ are presented in Fig. 1(a). The simulation shows that initially the indenter is far from the loading surface and does not interact with it. As the indenter approaches the crystallite, attractive force arises (the range of negative values in Fig. 1(a)) reaching its maximum at a distance of $3.0 \AA$ from the loaded surface. This "dip" on the curve of the crystallite reaction force involves bending of the loaded surface toward the indenter. This phenomenon is known as jump-to-contact [11]. The change of the loaded surface shape leads to generation of local structural changes in the crystallite region beneath the indenter. Structural analysis shows that atoms in this region have 12 nearest neighbors, but their surrounding corresponds to none of the known lattices.

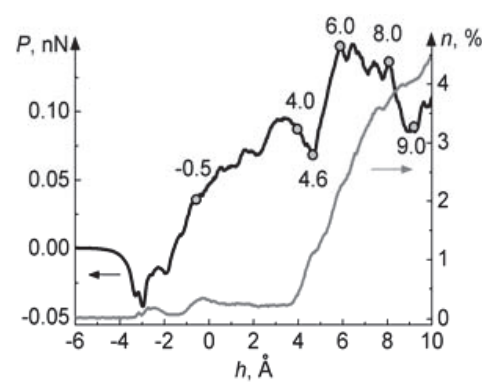

(a)

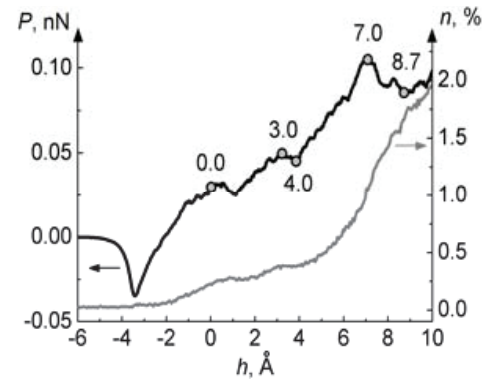

(b)

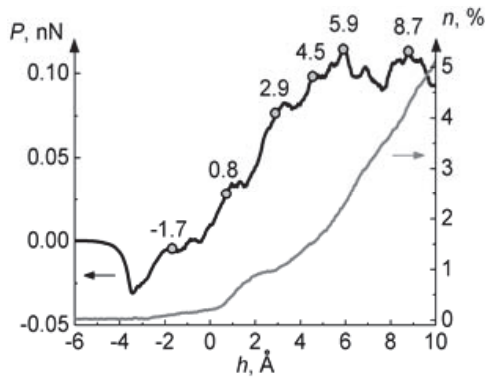

(c)

FIGURE 1. Crystallite reaction force (black curve) and fraction of atoms involved in structural changes (grey curve) vs indenter penetration depths for different loaded surface orientations: (a) (01 $\overline{1})$, (b) (001), (c) (111) 


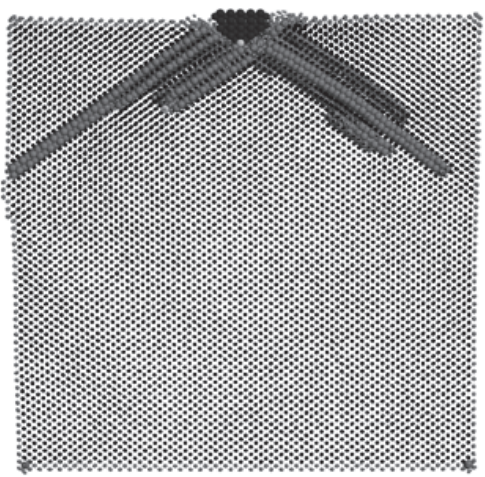

(a)

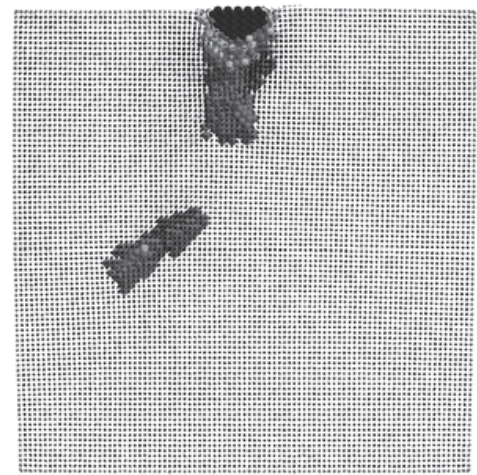

(b)

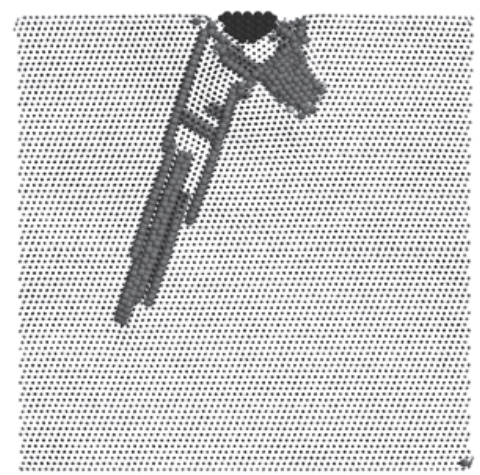

(c)

FIGURE 2. Crystallite structure fragments at an indenter penetration depth of $10.0 \AA$ for different loaded surface orientations: (a) $(01 \overline{1}),($ b) $(001),(c)(111)$. Atoms involved in local structural changes are presented as large grey spheres and atoms of indenter are in black

As the indenter moves further, the surface returns to its original position, the number of defects decreases, and the reaction force remains almost unchanged in the range from -2.5 to $-1.8 \AA$. Then, the reaction force increases with the number of defects kept constant. Once the point $-0.5 \AA$ is passed through, the slope of the curve decreases. This owes to an increase in the number of local structural changes which form partial dislocations $a / 6\langle 112\rangle\{111\}$ and then stacking faults. They are arranged in the adjacent atomic planes (11 $\overline{1})$ and $(1 \overline{1} 1)$. As the indenter moves further, the number of local structural changes decreases slightly and the dislocations remain immobile resulting in further growth of the crystallite reaction force. Beginning with an indentation depth of $3.6 \AA$, new stacking faults develop beneath the indenter in planes of the same type but somewhat below the earlier formed stacking faults which increase in size. As this takes place, the reaction force decreases greatly up to an indentation depth of $4.6 \AA$. The number of defects in further indentation continues to increase but the reaction force starts growing. This is likely because partial dislocations are detached from the indenter-crystallite contact zone and move toward the free surface. At an indentation depth of $6.0 \AA$, the reaction force peaks. As the dislocations reach the free surfaces, the crystallite changes its shape; in particular, jogs are formed on the free surface (Fig. 2(a)).

\section{Surface (001)}

The dependences of the reaction force and fraction of atoms involved in local structural changes on the indenter penetration depth for the loaded surface (001) are presented in Fig. 1(b). With the indenter positioned at $-3.4 \AA$, its attractive force to the surface peaks. The number of defects increases due to bending of the crystallite free surface. In further indentation, the reaction force and number of atoms involved in local structural changes begin to increase. Note that the reaction force curve reveals peculiar features at an indenter penetration depth of 0 and $3 \AA$. They are associated with the discrete indenter structure in which the distance between atomic rows in the indentation direction is $\sim 3 \AA$. As the indenter penetrates in the material, its atomic rows interact by turns with the crystallite. Because long-range atomic rows are initially attracted to the loaded surface, the growth of the crystallite reaction force slows down. Structural analysis of the model crystallite shows that at an indenter penetration depth of $-0.8 \AA$, the region formed in the contact zone contains atoms whose immediate surrounding has either hep symmetry or corresponds to none of the known lattices. The size of the region compares with the indenter size. In further indentation, the size of the defect region along the direction [001] increases with indenter penetration depth. Noteworthy is that more intense growth in the number of defects at an indentation depth of $7.0 \AA$ leads to a decrease in crystallite reaction force. The simulation shows that as the indenter stops at $10.0 \AA$, dislocation loops start moving from the vertex of the defect-containing region to the lateral faces of the crystallite (Fig. 2(b)). As the defects reach the free surfaces, jogs are formed and the fraction of local structural changes decreases from $2.0 \%$ to $0.3 \%$. 


\section{Surface (111)}

The dependences of the reaction force and fraction of atoms involved in local structural changes on the indenter penetration depth for the loaded surface (111) are presented in Fig. 1(c). The calculations show that local structural changes in the crystallite start developing at an indenter penetration depth of $-3.0 \AA$. They slow down the growth of the crystallite reaction force. At an indentation depth of $0.8 \AA$, the stacking fault in the plane (11 $\overline{1})$ starts growing and the reaction force changes little. In the adjacent planes $(11 \overline{1})$, generation and motion of a group of partial dislocations $a / 6\langle 112\rangle\{111\}$ takes place. As a result, a fragmented region bounded by microtwins with hep atoms located in twin planes is formed in the crystallite (Fig. 2(c)). It should be noted that in the indenter-crystallite contact zone, a fragmented region consisting of hcp atoms develops toward the right lateral face of the crystallite.

\section{CONCLUSION}

We studied the features of nucleation and development of plastic deformation in copper crystallites during nanoindentation with different crystallographic orientations of their loaded surface by molecular dynamics simulation. The results demonstrate that the response of the model crystallites depends largely on the loaded surface orientation. This shows up as differences in the sizes and orientations of damaged regions, types of structural defects, and crystallite reaction forces dependent on indentation depths.

\section{ACKNOWLEDGEMENTS}

The work was partially supported under program for competitiveness enhancement of Tomsk State University and Program of Basic scientific research of the State academies of sciences for 2013-2020.

\section{REFERENCES}

1. L. Wang, Z. Zhang, and X. Han, NPG Asia Mater. 5, 1 (2013).

2. C. A. Schuh, Mater. Today 9, 32 (2006).

3. I. Szlufarska, Mater. Today 9, 42 (2006).

4. S. G. Psakhie, K. P. Zolnikov, and D. S. Kryzhevich, Phys. Lett. A 367, 250 (2007).

5. S. G. Psakhie, K. P. Zolnikov, D. S. Kryzhevich, and A. G. Lipnitskii, Phys. Lett. A 349, 509 (2006).

6. S. G. Psakhie, K. P. Zolnikov, A. I. Dmitriev, D. S. Kryzhevich, and A. Yu. Nikonov, Phys. Mesomech. 15(34), 147 (2012).

7. M. I. Mendelev and A. H. King, Philos. Mag. 93, 1268 (2013).

8. M. S. Daw and M. I. Baskes, Phys. Rev. B 29, 6443 (1984).

9. J. D. Honeycutt and H. C. Andersen, J. Phys. Chem. 91, 4950 (1987).

10. H. V. Swygenhoven, D. Farkas, and A. Caro, Phys. Rev. B 62, 831 (2000).

11. U. Landman, W. D. Luedtke, N. A. Burnham, and R. J. Colton, Science 248, 454 (1990). 